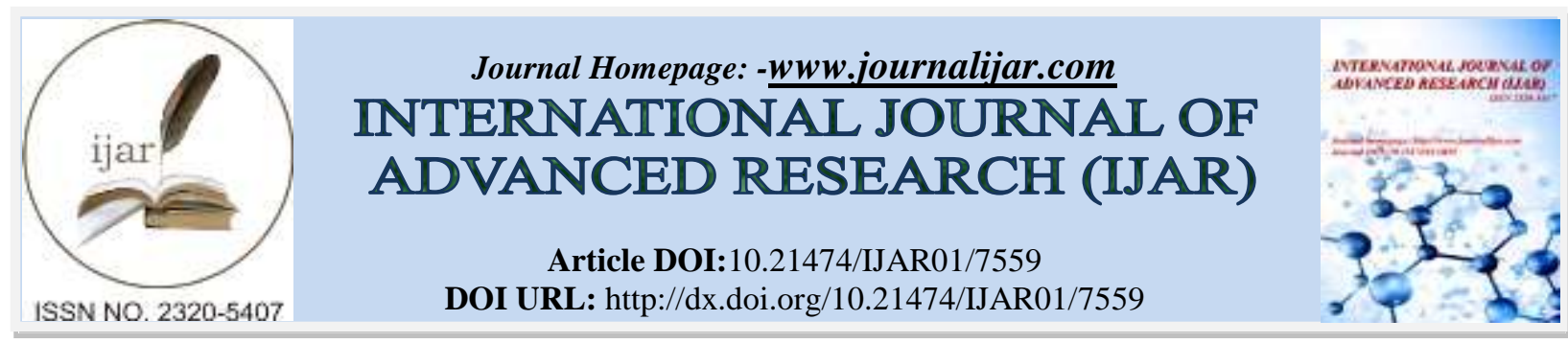

RESEARCH ARTICLE

\title{
FOLLICULAR OUTPUT RATE (FORT) MAY PREDICT INTRA CYTOPLASMIC SPERM INJECTION (ICSI) OUTCOME.
}

\author{
Hesham M. Fathy, Sherif F. El-Mekkawi ${ }^{1}$, Ahmed M. El-kotb ${ }^{2}$, Azza A. Abdel-Razek ${ }^{3}$, Amr A. Riad ${ }^{4}$ and \\ Omar G. Mustafa ${ }^{5}$.
}

1. Department of Obstetrics and Gynaecology.

2. Faculty of Medicine - Ain Shams University.

\section{Manuscript Info}

Manuscript History

Received: 13 June 2018

Final Accepted: 15 July 2018

Published: August 2018

Keywords:-

Follicualr output RaTe, FORT, ICSI, pre-ovulatory follicle count (PFC), ICSI outcome.

\section{Abstract}

Background: In IVF/ICSI treatment, basal (follicle stimulating hormone) FSH, antral follicle count (AFC) and serum anti-Mullerian hormone (AMH) concentrations are used to assess the basal ovarian reserves and to predict ovarian response and IVF/ICSI outcome. AFC; a non-invasive method, may well represent functional ovarian reserves and is well correlated to the number of oocytes retrieved. However, it cannot predict the oocyte /embryo quality or outcome in IVF/ICSI treatment. Aim of the Work: The aim of the study is to assess the accuracy and usefulness of the Follicular Output Rate (FORT); which was determined as a ratio of pre-ovulatory follicle count (PFC) on day of HCG administration to $(\mathrm{AFC}) \times 100$, as a predictor of pregnancy outcome after ICSI treatment cycles in infertile women. Patietns and Methods: This prospective cohort study was conducted on quota sampling used to recruit 100 infertile women undergoing ICSI in each of four study groups: polycyctic ovary syndrome (PCOS) group, tubal factor group, endometriosis group and unexplained infertility group, i.e. a total of 400 women were enrolled in the study in the Assisted Reproduction Unit (ART Unit) of Ain Shams University Maternity Hospital and private IVF centres in the period between February 2017 and July 2018. Results: PFC was significantly higher in the PCOS women compared to the other three groups (11.42 \pm 4.54 , $7.79 \pm 4.12,6.81 \pm 0.81$ and $7.53 \pm 2.61$ respectivily). PCOS women who achieved clinical pregnancy had significantly lower FORT than those who didn't $(0.56 \pm 0.21,0.66 \pm 0.19 ; \mathrm{P}=0.02)$. Tubal-factor women who achieved clinical pregnancy had significantly higher FORT than those who didn't $(0.66 \pm 0.25,0.53 \pm 0.26 ; \mathrm{P}=0.01)$. Endometriosis women who achieved clinical pregnancy had non significantly different FORT than those who didn't $(0.46 \pm 0.19,0.46 \pm 0.17 ; \mathrm{P}=0.99)$. Unexplained infertility women who achieved clinical pregnancy had significantly higher FORT than those who didn't $(0.62 \pm 0.19,0.53 \pm$ $0.15 ; \mathrm{P}=0.01)$. Conclusion: FORT may be a predictor of oocyte competence in terms of number of retrieved, mature and fertilized oocytes. The indicator can be used for prediction of clinical pregnancy rate after ICSI. The FORT predictive value, sensitivity, 
specificity and cut-off values to define poor ovarian responders and whether FORT can be used to determine when to cancel IVF/ICSI cycles for poor ovarian response are questions to be answered by further large scale research and meta-analysis which will undoubtfullycontribute to broaden its clinical applications.

Copy Right, IJAR, 2018,. All rights reserved.

\section{Introduction:-}

The health impact of infertility has resulted in aggravation of medical, social, psychological and economic burdens in developing countries ${ }^{(\mathbf{1})}$. Stress of infertility disturbs wellness of a couple in general and women in particular, resulting in emotional instability, distress and anxiety that may end up in depression ${ }^{(2)}$. The resolution to the issue is provided by assisted reproductive clinics where a number of treatment procedures are offered for the above. Intra Cytoplasmic Sperm Injection (ICSI) is one of the advanced techniques conceded after down regulation of ovaries, controlled Ovarian Stimulation (COS), Oocyte Pick Up (OPU) and micro injection of spermatozoa followed by Embryo Transfer (ET) $)^{(\mathbf{3 , 4})}$.

Intra cytoplasm sperm injection (ICSI) is a highly advanced technique of Assisted Reproductive Clinics (ARC) where conception does not occur by regular infertility treatment procedures. The technique although employed predominantly for male factors like compromised; sperm concentration, motility or morphology yet successful conception depends on oocyte parameters; like number of retrieved, mature and fertilized oocytes obtained as a result of treatment ${ }^{(\mathbf{3 , 4})}$.

Females have life time endowment of ovarian reserve (OR) at 20 weeks of her gestation when approximately 6-7 million oogonia are there in her ovaries. This number decreases to approximately 2-3 million at birth and then drops off to 300,000 at the time of puberty. After that, during reproductive years, ovary starts to recruit monthly at least 30-50 oocytes from ovarian reserve (OR).They compete with each other to become the dominant follicle and ultimately ovulate to release an egg for fertilization. A good reserve generally points toward a large number of viable oocytes and low OR represents smaller number of their availability. Diminished OR caused by increasing age is associated with decrease in number as well as quality of oocyte retrieved during assisted conception treatment ${ }^{(5)}$.

The likelihood of success depends on ovarian response to stimulation which is estimation of number of oocytes retrieved (NRO) from OR. Pre-treatment assessment of OR is made by ovarian reserve test (ORT) which gives an idea about probable response to ovarian stimulation ${ }^{(\mathbf{6})}$.

This knowledge is important in order to calculate the appropriate dose of stimulation with some information about quantity and quality of oocytes, as well as risk of cycle cancellation. Antral follicle count (AFC), a very common example and noninvasive method for determining OR refers to the total number of primordial follicles presents in both ovaries and suggests the responsiveness of antral follicles to $\mathrm{FSH}^{(4)}$.

COS in ICSI procedures employs recruitment of multiple follicles that grow and finally develop into mature follicles. Stimulation is achieved by administration of forms of exogenous gonadotropins which increase follicular recruitment, maturation of follicles and multiple eggs production ${ }^{(7)}$.

The attempt increases the number of oocytes for possibility of fertilization and cleavage of embryos available for transfer improving success rates. The number of oocytes retrieved can be predicted by several factors: especially female age, Antral Follicle Count (AFC), basal Follicle Stimulating Hormone (FSH) and Body Mass Index (BMI). ${ }^{(4)} \mathrm{AFC}$ represents number of remaining primordial pool in women and corresponds to the number of oocytes retrieved, however it does not influence oocyte/embryo quality and in turn outcome of ICSI. ${ }^{(\mathbf{8})}$.

The number of preovulatory follicles obtained at the end of COS at the same time is not a reliable reflection of antral follicle sensitivity to FSH, since it is subject to a number of small antral follicles available before treatment.

In this context, follicular output rate (FORT) has proved itself to be a qualitative reflector of ovarian follicular competence in terms of percentage of antral follicles that effectively respond to FSH after COS in ICSI patients. ${ }^{(9)}$. 
The need for estimation of FORT is hypothesized from the fact that follicle and oocyte dysfunction limit the sensitivity of antral follicles to exogenous FSH with the role of FORT as an important predictor of antral follicle responsiveness. This is especially useful to ignore $40 \%$ atretic follicles that might give false results to the expected stimulation $^{(\mathbf{1 0})}$.

\section{Aim of the Work:-}

The aim of the study is to assess the accuracy and usefulness of the Follicular Output Rate (FORT); the ratio between pre-ovulatory follicle counts (PFC) on day of HCG administration multiplied by 100 then divided by AFC at baseline, as a predictor of pregnancy outcome after ICSI treatment cycles in infertile women.

Patients and Methods:-

Study design:-Prospective cohort study.

\section{Settings:-}

The current study was conducted at the Assisted Reproduction Unit (ART Unit) of Ain Shams University Maternity Hospital and private IVF centres in the period between February 2017 and July 2018.

Population:Quota sampling was used to recruit 100 infertile women undergoing ICSI in each of four study groups: PCOS group, tubal factor group, endometriosis group and unexplained infertility group, i.e. a total of 400 women were enrolled in the study.

\section{Research Methodology:-}

After approval of the ethical committee of the obstetrics and gynecology department of Ain Shams University; all participants in the study were given a written, informed consent, after explaining the details of the study to them.

Participants included in this study had the following criteria:

a. Age: $18-39$ years.

b. BMI $\left(\mathrm{kg} / \mathrm{m}^{2):} 18-29\right.$

c. Normal office hysteroscopy.

d. Normal parameters of husband semen analysis according to WHO criteria 2010.

e. Written and signed informed consent by the patient to participate in the study.

The following patients were excluded from the study:-

Age less than 18 or more than 39 years. , Abnormal hysteroscopic finding, e.g. endometrial polyps, endometrial hyperplasia or fibroid., Abnormal ultrasonographic finding, e.g. endometrial polyps, fibroids or ovarian cysts., Abnormal endocrinological profile (apart from those attributed to PCOS), e.g. hypothyroidism, hyperprolactinemia., Abnormal male or female karyotyping., Abnormal semen analysis parameters according to WHO criteria 2010, Poor embryo quality in previous IVF trials., recurrent implantation failure, defined as failure to achieve a recognizable intrauterine gestational sac by ultrasonography after transfer of at least four good-quality embryos in a minimum of three fresh or frozen IVF cycles., Good-quality embryos will be defined as those characterized by absence of multinucleated blastomeres, four or five blastomeres on day 2 , seven or more cells on day 3 , and $\leq 20 \%$ anucleated fragments ${ }^{(11)}$, recurrent pregnancy loss, defined as loss of two or more consecutive pregnancies before 20 weeks gestational age, Positive serumanticardiolipin antibodies or lupus anticoagulant., Positive thrombophilia screen., any chronic medical disorder as cardiac, pulmonary or rheumatologic diseases., evidence of any other endocrine disorder such as diabetes mellitus, thyroid dysfunction, hyperprolactinemia, congenital adrenal hyperplasia, Cushing's syndrome or adrenal insufficiency., evidence of premature ovarian failure, i.e. persistently elevated or serially rising FSH levels, and mental condition rendering the patients unable to understand the nature, scope and possible consequences of the study.

\section{Definition of determinants of cohort allocation:-}

Patients fulfilling the inclusion and exclusion criteria were allocated to one of the four study cohorts:, Polycystic Ovary Syndrome (PCOS) Group, Tubal Factor Group, Endometriosis Group, Unexplained Infertility Group

All patients were subjected to the following:-

1. Pre-enrollment assessment

2. Controlled ovarian hyperstimulation and intracytoplasmic sperm injection - embryo transfer 


\section{Statistical analysis:-}

Statistical analysis was performed using Microsoft Excel $^{\circledR} 2010$ and statistical package for social sciences (SPSS ${ }^{\circledR}$ version 24.0). Data was prescribed as range, mean and standard deviation (for parametric variables), range, median and interquartile range (for non-parametric variables), and number and percentage (for categorical variables). Difference between variables of two groups was analyzed using student's t-test (for non parametric variables) and Chi-squared test (for categorical variables). Difference between variables of more than two groups was analyzed using one way ANOVA test (for parametric variables), Kuskal Wallis test (for non-parametric variables) and Chisquared test (for categorical variables). Correlation between two variables was estimated using Pearson's correlation coefficient (for parametric variables) and Spearman's rank correlation coefficient (for non-parametric variables). Significance level was set at 0.05 .

\section{Results:-}

No statistically significant differences were found between the four groups regarding age or duration of infertility; whereas a statistically significant difference was found between the four groups regarding body mass index. Tukey's post-hoc analysis (single-step multiple comparison procedure and statistical test) revealed statistically significant higher BMI in the PCOS group compared to the other three groups; whereas no differences were found on comparing the latter groups.

Also, a statistically significant heterogeneity in the type of infertility (primary/secondary) between the four groups was noted by chi-square testing

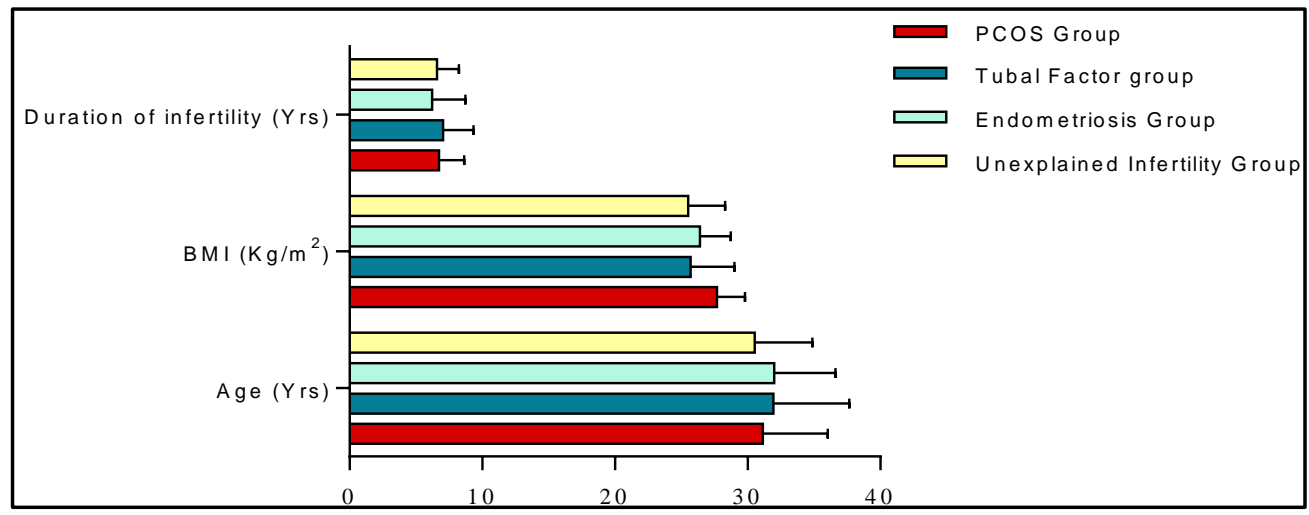

Figure 1:-Bar graph summarizes demographic and clinical data of the study groups.

Table 1:-Comparison between study groups regarding demographic and clinical characteristics

\begin{tabular}{|c|c|c|c|c|c|}
\hline & PCOS Group & $\begin{array}{c}\text { Tubal Factor } \\
\text { Group }\end{array}$ & $\begin{array}{c}\text { Endometriosis } \\
\text { Group }\end{array}$ & $\begin{array}{c}\text { Unexplained } \\
\text { Infertility Group }\end{array}$ & $\mathbf{P}$ \\
\hline $\begin{array}{l}\text { Age (years) } \\
\text { Range } \\
\text { Mean } \pm \text { SD }\end{array}$ & $\begin{array}{c}25.0-39.0 \\
31.14 \pm 4.89\end{array}$ & $\begin{array}{c}27.0-36.0 \\
31.94 \pm 5.71\end{array}$ & $\begin{array}{c}23.0-39.0 \\
32.01 \pm 4.62\end{array}$ & $\begin{array}{c}21.0-38.0 \\
30.52 \pm 4.37\end{array}$ & 0.14 \\
\hline $\begin{array}{l}\text { BMI }\left(\mathbf{K g} / \mathbf{m}^{2}\right) \\
\text { Range } \\
\text { Mean } \pm \text { SD }\end{array}$ & $\begin{array}{c}21.4-29.1 \\
27.68 \pm 2.11\end{array}$ & $\begin{array}{c}19.3-27.8 \\
25.71 \pm 3.29\end{array}$ & $\begin{array}{c}20.1-28.3 \\
26.41 \pm 2.31\end{array}$ & $\begin{array}{c}19.6-28.8 \\
25.52 \pm 2.78\end{array}$ & $<0.001$ \\
\hline $\begin{array}{l}\text { Type of infertility } \\
\text { Primary } \\
\text { Secondary }\end{array}$ & $\begin{array}{l}61(70.1 \%) \\
26(29.8 \%)\end{array}$ & $\begin{array}{c}6(6.3 \%) \\
89(93.6 \%)\end{array}$ & $\begin{array}{l}67(72.8 \%) \\
25(27.1 \%)\end{array}$ & $\begin{array}{c}82(95.3 \%) \\
4(4.6 \%)\end{array}$ & $<0.001$ \\
\hline $\begin{array}{l}\begin{array}{l}\text { Duration of infertility } \\
\text { (years) } \\
\text { Range } \\
\text { Mean } \pm \text { SD }\end{array} \\
\end{array}$ & $\begin{array}{c}2.0-11.0 \\
6.75 \pm 1.89\end{array}$ & $\begin{array}{c}3.0-10.0 \\
7.04 \pm 2.31\end{array}$ & $\begin{array}{c}2.0-9.0 \\
6.23 \pm 2.51\end{array}$ & $\begin{array}{c}2.0-9.0 \\
6.59 \pm 1.46\end{array}$ & 0.07 \\
\hline
\end{tabular}

Analysis of the IVF/ICSI Cycle Characteristics and Outcomes in the Study Groups:-

Analysis of basic IVF/ICSI cycle characteristics:-

Basal hormonal profile parameters differed significantly among the four study groups, as depicted by ANOVA analysis. Tukey's post hoc analysis revealed statistically significantly lower serum FSH in the PCOS and endometriosis groups compared to the tubal factor and unexplained infertility groups; whereas no differences were 
found between the former and latter groups. Serum LH was significantly higher in the PCOS group compared to the other three groups and higher serum LH in the endometriosis group compared to the unexplained infertility group; whereas no statistically-significant differences were found were found in any of the other pair-wise comparisons.

Antral follicular count was statistically significantly higher in the PCOS group compared to the other three groups; whereas no statistically significant differences were found between the latter three groups (as depicted by Tukey's post hoc testing).

$\mathrm{COH}$ regimen differed significantly among the four groups. Pair-wise comparisons of FSH total dose or duration of stimulation using Tukey's post hoc testing were all statistically significant, except when comparing PCOS and tubalfactor groups where the lower mean total dose of FSH and duration of stimulation in the PCOS women failed to reach statistical significance.

Table 2:-Comparison between study groups regards basic IVF/ICSI cycle characteristics

\begin{tabular}{|c|c|c|c|c|c|}
\hline & PCOS Group & $\begin{array}{c}\text { Tubal Factor } \\
\text { Group }\end{array}$ & $\begin{array}{c}\text { Endometriosis } \\
\text { Group }\end{array}$ & $\begin{array}{c}\text { Unexplained } \\
\text { Infertility Group }\end{array}$ & $\mathbf{P}$ \\
\hline $\begin{array}{l}\begin{array}{l}\text { Baseline } \\
\text { profile }\end{array} \\
\text { FSH } \\
\text { LH } \\
\mathrm{E}_{2}\end{array}$ & $\begin{array}{c}3.92 \pm 2.18 \\
9.54 \pm 1.88 \\
76.74 \pm 22.73\end{array}$ & $\begin{aligned} 5.31 & \pm 1.32 \\
3.13 & \pm 1.05 \\
40.89 & \pm 16.56\end{aligned}$ & $\begin{array}{c}4.11 \pm 1.74 \\
3.86 \pm 1.13 \\
49.76 \pm 13.74\end{array}$ & $\begin{array}{c}5.87 \pm 1.81 \\
2.93 \pm 1.21 \\
34.14 \pm 19.82\end{array}$ & $\begin{array}{l}<0.001 \\
<0.001 \\
<0.001\end{array}$ \\
\hline Antral follicular count & $19.70 \pm 5.95$ & $13.60 \pm 5.41$ & $14.99 \pm 3.87$ & $14.09 \pm 3.95$ & $<0.001$ \\
\hline $\begin{array}{l}\text { COH regimen } \\
\text { Duration of HMG (days) } \\
\text { Total dose of HMG (IU) }\end{array}$ & $\begin{array}{c}9.11 \pm 1.49 \\
1991.5 \pm 681.6\end{array}$ & $\begin{array}{c}9.12 \pm 1.11 \\
2179.29 \pm \\
609.2 \\
\end{array}$ & $\begin{array}{c}10.37 \pm 1.22 \\
2572.36 \pm 283.6\end{array}$ & $\begin{array}{c}11.96 \pm 1.39 \\
3244.56 \pm 364.1\end{array}$ & $\begin{array}{l}<0.001 \\
<0.001\end{array}$ \\
\hline
\end{tabular}

Analysis of follicular recruitment dynamics during IVF/ICSI cycle:-

Preovulatory follicular count was significantly higher in the PCOS women compared to the other three groups; whereas no statistically significant differences were found between the latter three groups as depicted by Tukey's post hoc testing.

No statistically significant differences in the follicular output rate (FORT) were found between the four study groups.

\section{Analysis of oocyte/embryo outcomes of IVF/ICSI cycle:-}

Number of oocytes retrieved was significantly higher in the PCOS group compared to the other three groups and lower in the endometriosis group compared to tubal-factor and unexplained infertility groups. No statistically significant differences were found between the latter two groups.

Number of MII oocytes was significantly higher in the PCOS group compared to the other three groups and also higher in the unexplained infertility group compared to endometriosis and tubal-factor groups. No statistically significant differences were found between the latter two groups.

Good quality embryo rate and clinical pregnancy rate differed significantly among the four study groups, being highest in the tubal-factor group and lowest in the unexplained infertility group.

Table 3:-Comparison between study groups regards follicular recruitment dynamics during IVF/ICSI cycle.

\begin{tabular}{|l|c|c|c|c|c|}
\hline & PCOS Group & $\begin{array}{c}\text { Tubal Factor } \\
\text { Group }\end{array}$ & $\begin{array}{c}\text { Endometriosis } \\
\text { Group }\end{array}$ & $\begin{array}{c}\text { Unexplained } \\
\text { Infertility Group }\end{array}$ & P \\
\hline $\begin{array}{l}\text { Preovulatory follicle } \\
\text { count }\end{array}$ & $11.42 \pm 4.54$ & $7.79 \pm 4.12$ & $6.81 \pm 0.81$ & $7.53 \pm 2.61$ & $<\mathbf{0 . 0 0 1}$ \\
\hline $\begin{array}{l}\text { Follicular output rate } \\
\text { (FORT) }\end{array}$ & $0.61 \pm 0.23$ & $0.59 \pm 0.21$ & $0.46 \pm 1.82$ & $0.56 \pm 0.15$ & 0.71 \\
\hline
\end{tabular}


Table 4:-Comparison between study groups regards oocyte/embryo outcomes from IVF/ICSI cycle.

\begin{tabular}{|l|c|c|c|c|c|}
\hline & PCOS Group & $\begin{array}{c}\text { Tubal Factor } \\
\text { Group }\end{array}$ & $\begin{array}{c}\text { Endometriosis } \\
\text { Group }\end{array}$ & $\begin{array}{c}\text { Unexplained } \\
\text { Infertility Group }\end{array}$ & P \\
\hline $\begin{array}{l}\text { Ovum pickup } \\
\text { No of oocytes retrieved }\end{array}$ & $9.88 \pm 2.12$ & $6.72 \pm 0.83$ & $5.93 \pm 0.89$ & $6.62 \pm 1.06$ & $<\mathbf{0 . 0 0 1}$ \\
No. of MII oocytes & $7.71 \pm 0.99$ & $4.17 \pm 0.89$ & $4.38 \pm 1.01$ & $5.46 \pm 1.09$ & $<\mathbf{0 . 0 0 1}$ \\
\hline $\begin{array}{l}\text { Good quality embryo rate } \\
(\%)\end{array}$ & $65.21 \%$ & $68.83 \%$ & $49.52 \%$ & $45.56 \%$ & $\mathbf{0 . 0 0 2}$ \\
\hline $\begin{array}{l}\text { Clinical pregnancy rate } \\
(\boldsymbol{\%})\end{array}$ & $50.57 \%$ & $53.68 \%$ & $42.39 \%$ & $40.69 \%$ & $\mathbf{0 . 0 4}$ \\
\hline
\end{tabular}

Analysis of the Effect of FORT and Other IVF/ICSI Cycle Characteristics on Pregnancy Outcome:Analysis of FORT and other IVF/ICSI cycle characteristics in PCOS women:-

No significant differences in AFC or $\mathrm{COH}$ regimen (either HMG total dose or duration of stimulation) between PCOS women who achieved clinical pregnancy and those who didn't. However, PCOS women who achieved clinical pregnancy had significantly lower preovulatory follicular count, FORT and number of retrieved oocytes; together with higher number of MII oocytes and good-quality embryo rate compared to women who failed to achieve clinical pregnancy.

Table 5:-Comparison between PCOS women who achieved clinical pregnancy and those who didn't; regarding follicular recruitment parameters and oocyte/embryo outcomes.

\begin{tabular}{|l|c|c|c|}
\hline & Clinical Pregnancy & No Clinical Pregnancy & P \\
\hline AFC & $19.67 \pm 5.98$ & $19.74 \pm 6.01$ & 0.95 \\
\hline COH regimen & & & \\
Duration of HMG (days) & $9.21 \pm 1.53$ & $9.01 \pm 1.46$ & 0.53 \\
Total dose of HMG (IU) & $1966.86 \pm 574.60$ & $2017.40 \pm 740.06$ & 0.72 \\
\hline Preovulatory follicle count & $10.56 \pm 4.07$ & $12.28 \pm 3.91$ & $\mathbf{0 . 0 4}$ \\
\hline Follicular output rate (FORT) & $0.56 \pm 0.21$ & $0.66 \pm 0.19$ & $\mathbf{0 . 0 2}$ \\
\hline Ovum pickup & & & \\
No of oocytes retrieved & $9.42 \pm 2.18$ & $10.35 \pm 2.11$ & $\mathbf{0 . 0 4}$ \\
No. of MII oocytes & $8.23 \pm 0.96$ & $7.19 \pm 1.16$ & $<\mathbf{0 . 0 0 1}$ \\
\hline Good quality embryo rate (\%) & $75.30 \%$ & $61.12 \%$ & $\mathbf{0 . 0 4}$ \\
\hline
\end{tabular}

Analysis of FORT and other IVF/ICSI cycle characteristics in tubal-factor women:-

No significant differences in AFC or COH regimen (either HMG total dose or duration of stimulation) between tubal-factor women who achieved clinical pregnancy and those who didn't. However, tubal-factor women who achieved clinical pregnancy had significantly higher preovulatory follicular count, FORT and number of retrieved oocytes, number of MII oocytes and good-quality embryo rate compared to women who failed to achieve clinical pregnancy.

Table 6:-Comparison between tubal-factor women who achieved clinical pregnancy and those who didn't; regarding follicular recruitment parameters and oocyte/embryo outcomes.

\begin{tabular}{|l|c|l|c|}
\hline & Clinical Pregnancy & No Clinical Pregnancy & P \\
\hline AFC & $13.86 \pm 5.36$ & $13.34 \pm 5.84$ & 0.65 \\
\hline COH regimen & & & \\
Duration of HMG (days) & $9.15 \pm 1.27$ & $9.10 \pm 1.35$ & 0.85 \\
Total dose of HMG (IU) & $2147.39 \pm 600.90$ & $2212.24 \pm 617.19$ & 0.60 \\
\hline Preovulatory follicle count & $8.42 \pm 2.90$ & $7.17 \pm 2.54$ & $\mathbf{0 . 0 2}$ \\
\hline Follicular output rate (FORT) & $0.66 \pm 0.25$ & $0.53 \pm 0.26$ & $\mathbf{0 . 0 1}$ \\
\hline Ovum pickup & & & \\
No of oocytes retrieved & $7.71 \pm 1.19$ & $5.74 \pm 0.66$ & $<.001$ \\
No. of MII oocytes & $5.24 \pm 0.89$ & $3.11 \pm 0.95$ & $<\mathbf{0 . 0 0 1}$ \\
\hline Good quality embryo rate (\%) & $75.77 \%$ & $55.90 \%$ & $\mathbf{0 . 0 0 4}$ \\
\hline
\end{tabular}


Analysis of FORT and other IVF/ICSI cycle characteristics in endometriosis women:-

No significant differences in AFC, $\mathrm{COH}$ regimen (either HMG total dose or duration of stimulation), preovulatory follicular count, FORT, number of retrieved oocytes, number of MII oocytes or good-quality embryo rate between endometriosis women who achieved clinical pregnancy and those who didn't.

Table 7:-Comparison between endometriosis women who achieved clinical pregnancy and those who didn't; regarding follicular recruitment parameters and oocyte/embryo outcomes.

\begin{tabular}{|l|c|c|c|}
\hline & Clinical Pregnancy & No Clinical Pregnancy & P \\
\hline AFC & $14.06 \pm 3.63$ & $15.52 \pm 4.02$ & 0.07 \\
\hline COH regimen & $10.12 \pm 1.16$ & $10.62 \pm 1.42$ & \\
Duration of HMG (days) & $2564.35 \pm 271.36$ & $2581.48 \pm 304.77$ & 0.07 \\
Total dose of HMG (IU) & $6.81 \pm 0.99$ & $7.21 \pm 1.22$ & 0.78 \\
\hline Preovulatory follicle count & $0.46 \pm 0.19$ & $0.46 \pm 0.17$ & 0.99 \\
\hline Follicular output rate (FORT) & & & \\
\hline Ovum pickup & $6.04 \pm 1.03$ & $5.83 \pm 0.72$ & 0.25 \\
No of oocytes retrieved & $4.56 \pm 1.02$ & $4.21 \pm 1.08$ & 0.11 \\
No. of MII oocytes & $51.12 \%$ & $47.92 \%$ & 0.67 \\
\hline Good quality embryo rate (\%) & & & \\
\hline
\end{tabular}

Analysis of FORT and other IVF/ICSI cycle characteristics in unexplained infertility women:-

No significant differences in AFC or $\mathrm{COH}$ regimen (either HMG total dose or duration of stimulation) between unexplained infertility women who achieved clinical pregnancy and those who didn't. However, unexplained infertility women who achieved clinical pregnancy had significantly higher preovulatory follicular count, FORT and number of retrieved oocytes, number of MII oocytes and good-quality embryo rate compared to women who failed to achieve clinical pregnancy.

Table 8:-Comparison between unexplained infertility women who achieved clinical pregnancy and those who didn't; regarding follicular recruitment parameters and oocyte/embryo outcomes.

\begin{tabular}{|l|c|c|c|}
\hline & Clinical Pregnancy & No Clinical Pregnancy & P \\
\hline AFC & $13.76 \pm 3.81$ & $14.42 \pm 4.62$ & 0.48 \\
\hline COH regimen & & & \\
Duration of HMG (days) & $11.92 \pm 1.42$ & $12.0 \pm 1.37$ & 0.79 \\
Total dose of HMG (IU) & $3217.62 \pm 293.53$ & $3272.74 \pm 488.59$ & 0.55 \\
\hline Preovulatory follicle count & $7.96 \pm 1.81$ & $7.11 \pm 1.62$ & $\mathbf{0 . 0 2}$ \\
\hline Follicular output rate (FORT) & $0.62 \pm 0.19$ & $0.53 \pm 0.15$ & $\mathbf{0 . 0 1}$ \\
\hline Ovum pickup & & & \\
No of oocytes retrieved & $7.02 \pm 1.05$ & $6.23 \pm 1.18$ & $\mathbf{0 . 0 0 2}$ \\
No. of MII oocytes & $5.91 \pm 1.01$ & $5.01 \pm 1.18$ & $\mathbf{0 . 0 0 4}$ \\
\hline Good quality embryo rate (\%) & $54.42 \%$ & $40.70 \%$ & $\mathbf{0 . 0 5}$ \\
\hline
\end{tabular}

\section{Discussion:-}

In IVF/ICSI treatment, basal FSH, AFC and serum anti-Mullerian hormone (AMH) concentrations are used to assess the basal ovarian reserves and to predict ovarian response and IVF/ICSI outcome.However, these indicators have several limitations. ${ }^{(11)}$.

AFC may well represent the actual functional ovarian reserves and is highly correlated to the number of oocytes retrieved ${ }^{(\mathbf{1 2})}$. However, it cannot predict the oocyte/embryo quality or the IVF outcome in an egg donation program (8).

Studies have shown that AMH could be a predictor of ovarian reserve and of IVF success ${ }^{(\mathbf{1 2})}$. However, conflicting research could not attribute predictive power of pregnancy outcomes to $\mathrm{AMH}^{(13)}$.

Similarly to most studies assessing the accuracy of predicting antral follicle sensitivity to FSH, PCOS cases were not excluded from the analyses. In fact, patients with PCOS have clearly elevated AFC and AMH concentrations (Broekmans et al., 2008; La Marca et al., 2009). 
The FORT is a measure represents the ratio of pre-ovulatory follicle count (PFC) on the day of human chorionic gonadotrophins (HCG) administration; to the antral follicle count (AFC) after complete suppression of endogenous gonadotrophins and it is independent of the preexisting antral follicle number. $($ FORT $=$ PFC/AFC $\times 100){ }^{(14)}$.

Clinical pregnancy rates are expected to be higher if a higher number of good quality embryos are available for transfer. High quality embryos are expected to be the result of high fertilization rate of good quality oocytes from responsive follicles.

When compared with poor quality follicles, high quality follicles are expected to be more responsive to FSH, thereby resulting in higher PFC which is an important measure of oocyte competence. An objective assessment tool of the follicular responsiveness to exogenous FSH; and consequently higher PFC will be of value in predicting IFV/ICSI outcome. The FORT can be this tool to achieve that goal and our study may be a little step forward.

To the best of our knowledge, the correlation between FORT and outcome in couples with different etiology of infertility undergoing IVF/ ICSI has not been investigated and compared in one study. Zhang et al. ${ }^{(11)}$ assessed the true accuracy of follicular output rate (FORT) as a prognostic indicator of response to FSH and reproductive competence after IVF/ICSI in PCOS patients and non PCOS patients.

The results of the current study show that Preovulatory follicular count (PFC) was significantly higher in the PCOS women compared to the other three groups $(11.42 \pm 4.54,7.79 \pm 4.12,6.81 \pm 0.81$ and $7.53 \pm 2.61$ respectivily); whereas no statistically significant differences were found between the latter three groups as depicted by Tukey's post hoc testing.

Our results agree with those of Zhang et al. (2013) as normal ovaries have fewer antral follicles and the saturation of the FSH receptor population may have limited estradiol production. In contrast, the PCOS ovary contains two- or three-times the number of antral follicles in a normal ovary. Women with PCOS exhibit significantly greater capacity for estradiol production in response to gonadotrophins stimulation as the result of a larger number of stimulated granulosa cells.

Good quality embryo rate and clinical pregnancy rate differed significantly among the four study groups, being highest in the tubal-factor group and lowest in the unexplained infertility group.

No significant differences in AFC or COH regimen (either FSH total dose or duration of stimulation) between PCOS women who achieved clinical pregnancy and those who didn't. However, PCOS women who achieved clinical pregnancy had significantly lower preovulatory follicular count, lower FORT value $(0.56 \pm 0.21$ versus $0.66 \pm 0.19$, respectively; $\mathrm{P}=0.02$ ) and lower number of retrieved oocytes; together with higher number of MII oocytes and higher number of good-quality embryos compared to women who failed to achieve clinical pregnancy.

Our results agree with those of Zhanget al. ${ }^{(11)}$ who concluded that women with PCOS who achieved clinical pregnancy exhibited significantly lower FORT values than those without clinical pregnancy and a better IVF/ICSI outcome was achieved in PCOS patients with medium FORT values.

Previous studies also had demonstrated the abnormalities of folliculogenesis and granulosa cell function in patients with PCOS. High estradiol concentrations on the day of HCG administration could potentially affect oocyte maturity and quality ${ }^{(15)}$.

Given the diverse clinical characteristics of infertile women with PCOS and their hyper responsiveness to FSH, it is important to moderate the dose of FSH to achieve a medium FORT value and thereby to improve the outcomes of IVF/ICSI in PCOS patients.

Our results show that there are no significant differences in AFC or $\mathrm{COH}$ regimen (either FSH total dose or duration of stimulation) between tubal-factor infertile women who achieved clinical pregnancy and those who didn't. However, tubal-factor women who achieved clinical pregnancy had significantly higher preovulatory follicular count, significantly higher FORT $(0.56 \pm 0.25$ versus $0.53 \pm 0.26$, respectively; $\mathrm{P}=0.01)$ and number of retrieved oocytes, higher number of MII oocytes and higher number good-quality embryo compared to women who failed to achieve clinical pregnancy. 
Zhang et al. (2013) results showed no difference in FORT between the pregnancy and non-pregnancy groups of nonPCOS patients but the rates of good-quality embryos, embryo implantations and clinical pregnancies increased dramatically in accordance with FORT values. The correlation analysis of that study indicated that FORT was correlated with the numbers of embryos suitable for the transfer of good-quality embryos. Zhang et al. (2013) concluded that in non-PCOS patients, FORT may be an indicator of response to FSH and of reproductive competence, as reflected by the outcome of oocytes and embryos after IVF/ICSI treatment. A better IVF/ICSI outcome was achieved in non-PCOS patients with high FORT values. Those conclusions in agree with our results.

The current study revealed no significant differences in AFC, COH regimen (either FSH total dose or duration of stimulation), preovulatory follicular count, FORT, number of retrieved oocytes, number of MII oocytes or goodquality embryo rate between infertile women with endometriosis who achieved clinical pregnancy and those who didn't.

Gallot et al. ${ }^{(9)}$ studied a cohort of infertile women (322) undergoing IVF/ICSI for male factor (40\%), tubal factor $(16 \%)$, endometriosis without ovarian lesions (10\%) and idiopathic $(25 \%)$; in $8 \%$ of cases, the same couple presented more than one of the IVF-ET indications listed above. They concluded that the average FORT was $50.6 \%$ (range, 16.7-100.0\%). Clinical pregnancy rates per oocyte retrieval increased progressively from the low to the high FORT groups $(33.3,51.2$ and $55.7 \%$, respectively, $\mathrm{P}, 0.003)$ and such a relationship assessed by logistic regression was independent of the confounding covariates, women's ages, AFC and PFC.

We found also no significant differences in the current study regarding AFC or COH regimen (either FSH total dose or duration of stimulation) between unexplained infertility women who achieved clinical pregnancy and those who didn't.

However, unexplained infertility women who achieved clinical pregnancy had significantly higher preovulatory follicular count, higher FORT and higher number of retrieved oocytes, higher number of MII oocytes and goodquality embryo rate compared to women who failed to achieve clinical pregnancy Genro et al. ${ }^{(\mathbf{1 4})}$ prospectively studied 179 (IVF-embryo transfer) candidates, 24-43 years of age with idiopathic infertility represented (36.4\%) of the studied population. It was merely observational and included only analysis of data. They concluded only that antral follicle responsiveness to FSH, as far as it is measurable by FORT, is negatively correlated to the circulating AMH levels in normo-cycling women. This association, which has been controlled for confounding covariates, is in keeping with the theory that AMH inhibits the sensitivity of antral follicles to FSH.

Hassan et al. ${ }^{(16)}$ conducted a study aimed to determine whether follicular output rate (FORT) can predict the clinical pregnancy rate in women with unexplained infertility undergoing IVF/ICSI. A total of 303 women with unexplained infertility, who were undergoing IVF/ICSI, were divided into three groups according to FORT values. There was a progressive and significant increase from the low to the high FORT groups in the clinical pregnancy rate (29.9\%, $43.3 \%$ and $57.8 \%$; $\mathrm{P}<0.001$ ), Multivariate logistic regression analysis revealed that the correlation between FORT and pregnancy was independent of potential confounding factors $(\mathrm{P}=0.008)$.

In agreement with our study; Hassan et al. ${ }^{(16)}$ found that higher FORT values had better oocyte yield and clinical pregnancy rates in women with unexplained infertility undergoing IVF/ICSI with potentially normal ovarian response. They found that the number of excellent quality embryos was significantly lower in the low FORT group.

As a whole, the present study results agree with previous researches done by Gallotet al. ${ }^{(9)}$ and Zhang et al. (11). Gallotet al. ${ }^{(9)}$ studied the role of FORT in 322 subfertile women and Zhang et al. (2013) studied the role of FORT in 1503 subfertile women without PCOS in addition to 140 women with PCOS undergoing IVF/ICSI. Both studies found that the retrieved oocytes, total number of embryos, proportion of good quality embryos and pregnancy rate increased progressively from the low to the high FORT groups.

The significance of our results should take into consideration some limitations of FORT calculation, as discussed elsewhere in previous researches. (Genro et al.,2011).

FORT could not be estimated in patients who discontinued FSH treatment before hCG administration (due to a markedly insufficient follicle recruitment), since their PFC could not be established. Further studies are needed to verify if FORT still is predictive when the number of average-sized follicles at the mid-follicular phase of $\mathrm{COH}$ is 
used instead of the 16-22 mm follicles on day of HCG. Such a development might facilitate incorporation of patients prone to cycle cancellation into the FORT calculation.

In addition, FORT implies that 3-8 $\mathrm{mm}$ follicles before $\mathrm{COH}$ respond coordinately to $\mathrm{FSH}$, which is not always the case ${ }^{(17)}$. To overcome this limitation, the individual tracking of the development of each follicle in response to FSH would be required, which is practically unrealistic.

Furthermore, FORT assumed that, on day of HCG, only16-22 mm follicles effectively responded to FSH, while it is conceivable that smaller follicles also presented some degree of FSH responsiveness. However, as very small follicles, which were not countable by ultrasound at baseline, may have also initiated their FSH-driven maturation after the start of $\mathrm{COH}$ and reached intermediate sizes on day of $\mathrm{HCG}$, the inclusion of average-sized follicles on day of hCG into the calculation of FORT could confuse its interpretation.

Future evaluation of alternative ways of calculating the FORT, in particular using different numerator and including patients treated with weaker and possibly more discriminating exogenous FSH signals, will undoubtedly contribute to broaden its clinical applications.

\section{Conclusion:-}

FORT may be a predictor of oocyte competence in terms of a number of retrieved, mature and fertilized oocytes. The indicator can be used for prediction of clinical pregnancy rate after ICSI. It also gives information about the number of cleaved embryos and clinical pregnancy rate which needs to be further explored.

The FORT predictive value, sensitivity, specificity and cut-off values to define poor ovarian responders and whether FORT can be used to determine when to cancel IVF/ICSI cycles for poor ovarian response are questions to be answered by further large scale research and meta-analysis.

Moreover, additional studies focusing on other follicle sizes will be helpful to fine-tune alternative relevant cutoffs for the calculation of this new parameter. Together, these FORT characteristics bring out its great flexibility and spurs to further develop this new concept. Future evaluation of alternative ways of calculating the FORT, in particular using different numerator sand including patients treated with weaker and possibly more discriminating exogenous FSH signals, will undoubtedly contribute to broaden its clinical applications

\section{Recommendations:-}

Further clinical and basic research on a large scale number of patients is needed to understand the relationship between FORT and different parameters of ovarian follicles competence and the predictive value of FORT in relation to the IVF/ICSI outcome.

\section{References:-}

1. Rijal B, Shrestha R, Jha B. Association of thyroid dysfunction among infertile women visiting infertility center of Om Hospital, Kathmandu, Nepal. Nepal Med Coll J. 2011;13(4):247-9.

2. Shaheen R, Subhan F, Sultan S, SubhanKh, Tahir F. Prevalence of infertility in a cross section of Pakistani population. Pakistan J Zool. 2010; 42(4): 389-393

3. Rehman R, Hussain Z, SiddiqAA.Role of Progesterone in human embryo implantation. Rawal Med J 2012; 37(2): 194-198

4. Rehman R, Hussain Z, ZuberiNA.Prediction of success in intracytoplasmic sperm injection (ICSI) by estimation of serum Estradiol/Progesterone ratio on the day of embryo transfer. J Pak Med Assoc 2013; 63(5): 609-613

5. Toner JP.Ovarian reserve, female age and the chance for successful pregnancy. Minerva Gincecol 2003; 55(5): 399-406.

6. Hendriks DJ, Mol B-WJ, Bancsi LF, TeVelde ER, Broekmans FJ.Antral follicle count in the prediction of poor ovarian response and pregnancy after in vitro fertilization: a meta-analysis and comparison with basal follicle-stimulating hormone level. FertilSteril 2005; 83(2): 291-301

7. Toporcerova S, Adam J, Toporcer T, Hredzak R, Ostro A. Basal concentrations of FSH (follicle stimulating hormone) as a transfer outcome. Hum Reprod. 2006;27(4):1066-72. 
8. Melo MA, Garrido N, Alvarez C, Bellver J, Meseguer M, Pellicer A, Remohı' J.Antral follicle count (AFC) can be used in the prediction of ovarian response but cannot predict the oocyte/embryo quality or the in vitro fertilization outcome in an egg donation program. FertilSteril 2009;91:148-156.

9. Gallot V, Berwanger da Silva AL, Genro V, Grynberg M, Frydman N, Fanchin R.Antral follicle responsiveness to follicle-stimulating hormone administration assessed by the Follicular Output RaTe (FORT) may predict in vitro fertilization-embryo transfer outcome. Hum Reprod. 2012;27(4):1066-72.

10. Gougeon A. Regulation of ovarian follicular development in primates: facts and hypotheses. Endocr Rev. 1996;17(2):121-55.

11. Van Royen E, Mangelschots K, De Neubourg D, Valkenburg M, Van de Meerssche M, Ryckaert G, Eestermans W, Gerris J. Characterization of a top-quality embryo, a step towards single-embryo transfer. Hum Reprod1999; 14:2345-2349

12. Zhang N, Hao CF, Zhuang LL, Liu XY, Gu HF, Liu S, Chen ZJ. Prediction of IVF/ICSI outcome based on the follicular output rate.Reproductive biomedicine online. 2013 Aug 1;27(2):147-53.

13. Broer SL, Mol BW, Hendriks D, Broekmans FJ. The role of antimullerian hormone in prediction of outcome after IVF: comparison with the antral follicle count. FertilSteril 2009;91:705-714.

14. T. H. Lee, C. H. Liu, C. C. Huang, K. C. Hsieh, P. M. Lin, and M. S. Lee, Impact of female age and male infertility on ovarian reserve markers to predict outcome of assisted reproduction technology cycles, Reproductive Biology and Endocrinology, vol. 7, article 100, 2009.

15. Genro VK, Grynberg M, Scheffer JB, Roux I, Frydman R, Fanchin R. Serum anti-Mullerian hormone levels are negatively related to Follicular Output RaTe (FORT) in normo-cycling women undergoing controlled ovarian hyperstimulation. Hum Reprod 2011;26:671-677.

16. Aboulghar M. Prediction of ovarian hyperstimulation syndrome (OHSS). Estradiol level has an important role in the prediction of OHSS. Hum Reprod 2003;18:1140-1141.

17. Hassan A, Kotb M, AwadAllah A, Wahba A, Shehata N. Follicular output rate can predict clinical pregnancy in women with unexplained infertility undergoing IVF/ICSI: a prospective cohort study. Reproductive biomedicine online. 2017 Jun 1;34(6):598-604.

18. Fanchin R, Schona"uer LM, Cunha-Filho JS, Me'ndez Lozano DH, Frydman R. Coordination of antral follicle growth: basis for innovative concepts of controlled ovarian hyperstimulation. SeminReprod Med 2005;23:354-362. 